\title{
¿Es exportable la flexiguridad? Un estudio comparado de Italia y España*
}

\author{
Laura LEONARDI \\ Università degli Studi di Firenze \\ Laura.Leonardi@unifi.it \\ Antonio MARTÍN ARTILES \\ Universidad Autónoma de Barcelona, QUIT-IET \\ Antonio.Martin@uab.es \\ Óscar MOLINA \\ Universidad Autónoma de Barcelona, QUIT-IET \\ Oscar.Molina@uab.es \\ Davide CALENDA \\ Università degli Studi di Firenze \\ Davide.Calenda@unifi.it \\ Pilar CARRASQUER OTO \\ Universidad Autónoma de Barcelona, QUIT-IET \\ Pilar.Carrasquer@uab.es
}

Recibido: 12-11-2009

Aceptado: 25-10-2010

\begin{abstract}
RESUMEN
En este artículo analizamos el concepto de flexiguridad y su implementación en el Sur de Europa. Interesa mostrar los diferentes significados y variedades de flexiguridad, a través de comparar España e Italia. A partir de ahí, cuestionamos la idea de la transposición del modelo danés/holandés de flexiguridad al resto de países europeos, en particular a los del "modelo mediterráneo". Es decir, cuestionamos la viabilidad de transponer modelos mediante métodos de gobernanza multinivel que aspiren a una cierta convergencia inducida desde la UE. La variedad de modelos sociolaborales europeos hace que la flexiguridad tenga significados y manifestaciones distintas, incluso en casos similares como España e Italia, donde los distintos contextos pautan divergencias significativas a corto plazo en sus patrones de flexiguridad, aunque ello pueda matizarse si adoptamos un arco temporal más amplio. Así, el artículo muestra, para esos dos países, cómo el efecto societal limita la influencia del efecto inducido.
\end{abstract}

Palabras clave: Flexiguridad, políticas de empleo, empleabilidad, efecto societal, efecto inducido.

* Este artículo es el resultado de un estudio comparado entre Italia y España, realizado en el marco del Programa de Acción Integrada financiado por los Ministerios de Educación de ambos países (Ref. HI2007-0104). 


\title{
Can Flexicurity be Exported? A Comparative Study of Italy and Spain
}

\begin{abstract}
This paper approaches the issue of flexicurity and its implementation in Southern Europe. The comparison between Italy and Spain shows the existence of different meanings and varieties of flexicurity. Hence we question the transposition of the Dutch / Danish flexicurity model to other European countries, and more specifically to those belonging to the Mediterranean cluster. In other words, we wonder about the viability of transposing employment models through multi-level governance mechanisms that aim at some convergence across countries. The variety of socio-economic models explains the existence of different meaning and manifestations of flexicurity even between the most-similar cases of Italy and Spain where short-term developments have pointed towards divergence in their flexicurity equilibriums, though the picture changes if we adopt a long-term perspective. The article shows how the societal effect limits the influence of the induced EU effect.
\end{abstract}

Key words: Flexicurity, European labour policies, Employment protection , Unemployment benefits, Active labour policies.

\section{REFERENCIA NORMALIZADA}

Leonardi, L., Martín Artiles, A., Molina, O., Calenda, D., Carrasquer Oto, P (2011). ¿Es exportable la flexiguridad? Un estudio comparado de Italia y España. Cuadernos De Relaciones Laborales Vol. 29, núm.2, p. 417-443.

SUMARIO: 1. Introducción. 2. El concepto de flexiguridad 3. Flexibilidad en el mercado laboral. 4. Las prestaciones por desempleo. 5. Las políticas activas de empleo. 6. Conclusiones. 7. Bibliografía

\section{Introducción}

El objetivo de este artículo es analizar el modelo de flexiguridad que nace de las experiencias políticas de los países nórdicos y su posible traslación al sur de Europa, en particular a España e Italia. Ese modelo ha sido tomado desde la Unión Europea como paradigma para conjugar la flexibilidad del mercado laboral y el mantenimiento de un modelo social europeo que provea unos niveles mínimos de seguridad. $\mathrm{O}$, en un sentido más amplio, para garantizar la flexibilidad de los mercados sin menoscabo de la cohesión social. Nuestra hipótesis es que, en términos económicos, políticos e institucionales, esa experiencia nórdica de flexiguridad está alejada de los países del sur de Europa, de modo que su transposición se antoja cuanto menos problemática. Ni siquiera dentro del marco de implementación flexible que ofrece el Método Abierto de Cooperación como mecanismo de la gobernanza multinivel europea. Para contrastar esa hipótesis comparamos la experiencia de Italia y España: ambos países tienen características económicas, sociales y culturales muy parecidas, que precisamente los diferencian de los países del norte de Europa. De hecho, algunos analistas (Banyuls et alii, 2009) ya han planteado la existencia de un "modelo de empleo del Sur de Europa", además de un "modelo latino de relaciones laborales", así como la idea de un "modelo mediterráneo de bienestar" (Ferrera, 2005; Amable, 2003), a tenor de una serie de características compartidas por esos países, en especial, entre España e Italia. 
Bajo este prisma hemos abordado el estudio de la flexiguridad. Discutimos, en primer lugar, el propio concepto de flexiguridad y algunos rasgos generales de su implementación en Europa a través de mecanismos de gobernanza multinivel. Después analizamos las tres principales dimensiones de la flexiguridad (flexibilidad del mercado laboral, prestaciones por desempleo y políticas activas de empleo) en España e Italia. La última sección concluye analizando la situación y evolución de las políticas asociadas con la flexiguridad a la luz de los diversos contextos sociales y políticos así como las directrices europeas en los dos países objeto de estudio.

Como resultado, nuestro análisis muestra como la implementación o importación del paradigma de flexiguridad produce resultados dispares en los dos países analizados, y en cualquier caso alejados de los equilibrios institucionales que se observan en países como Holanda y Dinamarca. En las últimas dos décadas, Italia y España han encarado de modo diverso la tensión entre flexibilidad y seguridad, fruto de la disparidad en sus respectivos contextos socio-productivos, institucionales y políticos. Aunque también parecen influir factores de más largo recorrido, como las tradiciones de regulación y de acción colectiva, así como los cambios en la relación estado-mercado-familia en términos de provisión de seguridad y de bienestar, o de distribución del riesgo y de gestión de la incertidumbre, en cada país. Esas diferencias no impiden la existencia de rasgos convergentes, sobre todo en lo que respecta a cómo afrontan el impacto de la última crisis, en un contexto caracterizado por unos mercados laborales fuertemente segmentados y segregados, en ambos países.

\section{El concepto de flexiguridad}

El concepto de flexiguridad, que nace de las directrices de la política de empleo de la Unión Europea, invita a los Estados miembros a reformar sus mercados de trabajo y sus políticas sociales para combinar una flexibilidad en el mercado laboral que no se cuestiona, con una provisión de seguridad que garantice la cohesión social. El problema de fondo que ha justificado la adopción de éste paradigma estriba en la creciente inseguridad derivada de las formas atípicas de empleo, como el empleo temporal y el empleo a tiempo parcial, y su fuerte extensión en todos los países de la Unión Europea desde la década de los noventa. Unas formas de empleo que, como novedad, tienden a afectar no sólo a los colectivos habituales (mujeres y/o jóvenes) sino también al núcleo central de la mano de obra. De ahí la preocupación por el nexo entre flexibilidad y seguridad que se ha venido reflejando en sucesivos documentos de la Comisión Europea. Así, aparece ya en 1993, en el Libro Blanco "Crecimiento, competitividad y empleo" y un año más tarde se menciona en la Cumbre de Essen. También en la Cumbre de Ámsterdam de 1997 y, ese mismo año, en el Libro Verde, "Cooperación para una nueva organización del trabajo", que sugería mantener un equilibrio entre flexibilidad y seguridad. Posteriormente, en la Cumbre de Lisboa (2000) emerge como un aspecto de la 
Estrategia Europea de Empleo. Finalmente, el Informe de Wim Kok (European Commission 2004) concluía que "el reto para el mercado de trabajo consiste en encontrar un equilibrio justo entre la flexibilidad y la seguridad." Más recientemente encontramos el término de flexiguridad en las Líneas Directrices Integradas para el crecimiento y el empleo para el ciclo 2005-2007.

El concepto de flexiguridad se inspira en la experiencia de coordinación de las políticas activas de empleo, la política de formación y las medidas en materia de seguridad por desempleo en Dinamarca y Holanda. La experiencia danesa, en particular, constituye un modelo de empleo y seguridad, quizás único. La legislación danesa tiene un bajo nivel de protección del empleo, permitiendo a los empresarios el despido de trabajadores sólo con un breve tiempo de pre-aviso (3 días en muchos casos), de modo que el mercado laboral danés funciona de manera muy similar al de los países liberales (Reino Unido, Irlanda, Canadá y Estados Unidos). De hecho, se puede calificar el caso danés como un modelo de flexibilidad cuantitativa, como lo pone de relieve el indicador de la rotación del empleo, que ha venido oscilando en los últimos años entre el 25\% y el 30\% (Madsen, 2004:191). Como contrapartida, cuenta con un sistema de protección del desempleo alto, que cubre el $90 \%$ del salario del desempleado durante un año y puede prorrogar el seguro de desempleo hasta cuatro años, un sistema de formación que ofrece muchas oportunidades para la mano de obra desempleada, además de otras medidas de protección social, en el marco de un estado de bienestar fuerte y una economía con una fuerte orientación exportadora.

La flexiguridad así entendida, ha fascinado a las autoridades políticas de la Unión Europea. La consideran como un modelo interesante y viable para el conjunto de países europeos. Interesante porque ese modelo responde a una especie de "triangulo mágico" que combina tres elementos: 1) un mercado laboral flexible numéricamente, que facilita la transición de entrada, salida y reincorporación al mercado laboral; 2) el apoyo a la transición a una actividad laboral distinta a través de importantes programas de formación y reciclaje profesional, como parte de las políticas activas de empleo ${ }^{1}$; y 3 ) un sistema de solidaridad plasmado en una generosa provisión pública de pensiones de protección contra el desempleo. Y viable porque, en clave jurídica y desde planteamientos neo-funcionalistas, se considera que la "modernización" del derecho laboral y la adopción de pautas políticas comunes a nivel europeo tarde o temprano "inducen" la convergencia entre distintos

${ }^{1}$ Un indicador de la importancia que tiene la educación, formación continua y el reciclaje de la población adulta en Dinamarca es que en el año 2006, el 29,2 \% de los ocupados comprendidos entre los 25 y 64 años participan en cursos de formación. Comparado con otros países se pueden observar las diferencias: en España la participación es sólo del 10,4\%; en Francia, 7,5\%; en Italia, 6,1\%. La media de la UE-15 es del 11,1\%. Se entiende que, en el caso danés, el aumento de la participación de los trabajadores adultos en la formación ha constituido una respuesta a las políticas de reestructuración e innovación tecnológica. Esta participación ha venido en aumento desde 1995. Véase Eurostat (2007). 
modelos sociales (Scharpf, 1999). La flexiguridad se ha convertido en el principio inspirador de la Estrategia Europea de Empleo que se implementa a través del Método Abierto de Coordinación como mecanismo de gobernanza multinivel. Según éste último, la UE debe limitarse a fijar una serie de objetivos políticos y a proveer una serie de buenas prácticas que sirvan para orientar las políticas de los respectivos países. Contrariamente al método tradicional de regulación fuerte a través de directiva, en el caso de las políticas europeas gobernadas según el método abierto, las autoridades nacionales gozan de altos niveles de autonomía para decidir acerca de los mecanismos necesarios para alcanzar los objetivos establecidos. Es por esto que hablamos de transposición del modelo danés de flexiguridad al resto de países, a través de su adopción como paradigma que guía las políticas europeas de empleo.

Gráfico 1. El modelo de flexiguridad danesa

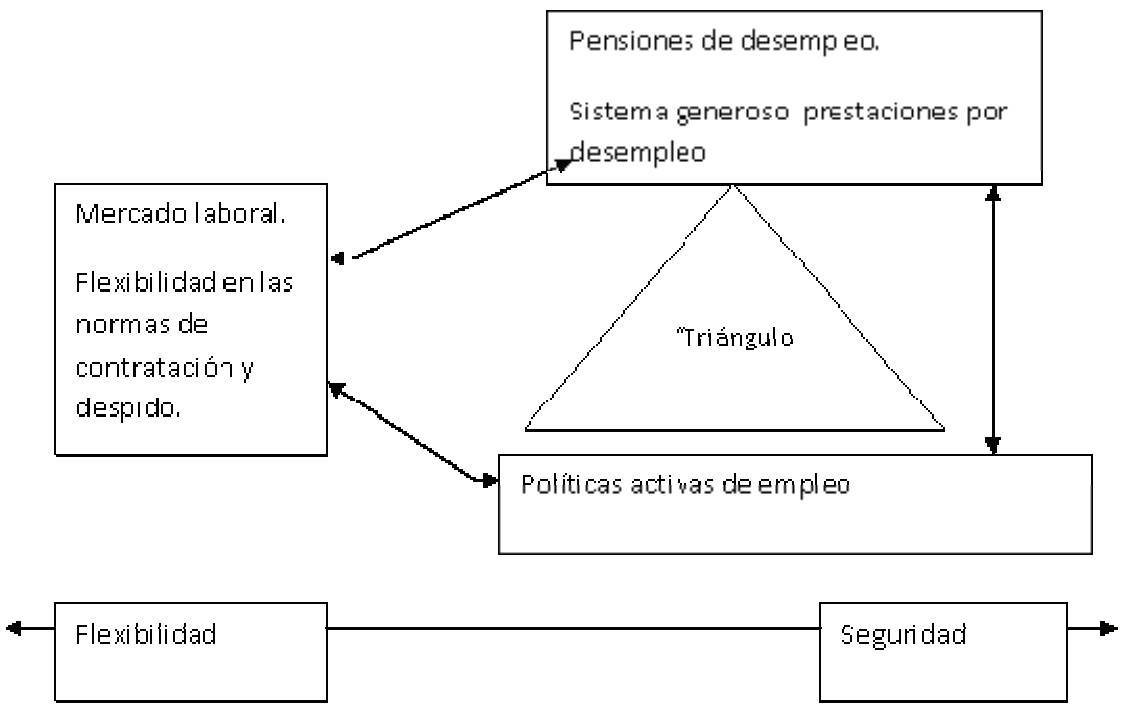

Pese a su entusiasta adopción por la Comisión Europea y su rápida extensión, el concepto de flexiguridad ha sido objeto de diversas críticas tanto desde el plano académico (para una síntesis véase Miguélez, 2007; Seifert y Tangian, 2007; Keune y Jepsen, 2007; Jepsen, 2005), como desde el plano ideológico o político donde un número creciente de voces cuestiona su idoneidad. La primera fuente de confusión y crítica deriva directamente del carácter polisémico de un concepto utilizado para 
indicar tanto una estrategia europea, como un sistema socio-institucional o modelo social, o, simplemente, como una herramienta heurística para el análisis de los mercados de trabajo europeos (Madsen 2006; Barbier, 2007).

En el primer caso, la flexiguridad se entiende como una estrategia que pretende coordinar las políticas de mercado de trabajo, con las políticas de prestaciones por desempleo y los programas de formación continua para favorecer la movilidad y las transiciones laborales. La diversidad de equilibrios que pueden darse como resultado de ésta coordinación refleja la ambigüedad de ese concepto. Algunos analistas como Rogowski (citado en Keune y Pochet, 2008:141), sostienen que, en realidad, la flexiguridad es una fuente de inspiración abstracta e inclusiva para elaborar determinadas políticas correctoras del mercado. Pero de igual forma, el mismo concepto ha sido empleado para legitimar políticas de flexibilización de los mercados laborales. En realidad, sería gracias a su inclusividad y vaguedad, que la flexiguridad puede convertirse en una aspiración de tipo ideal capaz de guiar las decisiones políticas y orientar la coordinación de las políticas públicas, con la finalidad de que las instituciones regulen y corrijan los desequilibrios del mercado y, al mismo tiempo, se implementen políticas que aumenten la flexibilidad en los mercados de trabajo.

Desde el punto de vista de la regulación de la actividad laboral, la "flexiguridad" constituye el propósito del Libro Verde de la Comisión Europea (2006), que se presenta con la idea de "Modernizar el Derecho Laboral para afrontar los retos del siglo XXI." El sesgo ideológico del documento, puede resumirse en los puntos que sugieren Baylos y Pérez Rey (2006). Primero, se culpabilizaría al Derecho laboral de que su sistema de derechos y garantías de protección es una tradición del pasado que induce hoy a "rigideces normativas". Por lo que habría que proceder a desregular y flexibilizar los elementos básicos del derecho para propiciar un mayor dinamismo al mercado laboral. La clave del argumento es que la desregulación aceleraría los flujos de entrada al mercado laboral, de manera que el derecho se orienta hacia el "empleo" y no a la protección de las condiciones de trabajo y a las garantías del mismo. Como señalaba Supiot (2003), el principio tuitivo y moral del derecho laboral estaría siendo sustituido por una visión "técnica" de mínimos. Segundo, el propósito del Libro Verde sería "descolectivizar" el derecho del trabajo mediante la exclusión de los actores colectivos, como son los sindicatos y los mecanismos de representación colectiva de los trabajadores en la empresa. El sujeto contractual principal sería el individuo, que a su vez es responsable de su "empleabilidad", en buena medida a través de su compromiso al reciclaje profesional continuo. Y, tercero, la negociación colectiva sería vista, de hecho, como una función meramente reguladora y normativa concurrente con la norma estatal y no como un proceso de intercambio y de diálogo entre sujetos colectivos para la distribución de posiciones de poder dentro y fuera de la empresa. La política neoliberal de la UE trataría de evitar el papel de la acción colectiva como correctora de las desigualdades a través de la negociación colectiva y el pacto social, limitándola sólo a su función reguladora dentro de la empresa. 
De lo anterior se desprende que la flexiguridad como paradigma para la regulación del mercado de trabajo resulta extremadamente ambiguo: oscila, por un lado, entre un "tipo ideal" orientador de posibles políticas de seguridad y bienestar, compensadoras de la precariedad del empleo e incluso protectoras del desempleo, hasta, por otro lado, un argumento para instrumentalizar un nuevo discurso ideológico a favor de una mayor desregulación y flexibilización del mercado laboral. Los actores sociales tampoco han logrado consensuar su contenido porque la idea de flexiguridad tiene distintos significados, en función del contexto histórico e institucional de la sociedad en cuestión. Es más, frente a la explosión de análisis cuantitativos de la flexiguridad que se ha producido en los últimos años, el concepto en si permanece vagamente teorizado ya que gran parte de la discusión se ha centrado en las virtudes de los modelos danés y holandés, comparándolas, sin más, con los resultados en otros países.

En este artículo proponemos re-considerar el concepto de flexiguridad tanto desde la perspectiva de los inputs (la visión de flexiguridad como modelo para orientar políticas) como desde la perspectiva de los resultados. Así pues consideramos la flexiguridad como un marco cognitivo (Barbier, 2007) a través del cual puede identificarse e interpretarse las interacciones entre ciertas características del mercado laboral y del sistema de protección social, en un determinado contexto sociopolítico, entendiendo este último como un entramado cultural que define las relaciones entre grupos sociales, o lo que es aceptable de lo que no, en términos de políticas y de empleo. Asimismo, desde el punto de los outputs, creemos adecuado considerar la flexiguridad como el resultado en políticas de la interacción entre diferentes mecanismos de gobernanza de la incertidumbre (Crouch 2008). Desde éste punto de vista, los resultados que se observan en los modelos danés u holandés, sólo podrían darse en el marco de un tipo concreto de gobierno de la incertidumbre: el de la "nueva democracia social".

Tanto si consideramos la flexiguridad como un marco cognitivo, como si nos centramos en las formas de gobernanza de la incertidumbre, su práctica real y efectiva está sujeta a las contingencias institucionales, económicas y políticas de los contextos domésticos. Así pues, a la hora de explicar los diferentes equilibrios que observamos al analizar casos reales, es importante tener en cuenta que la flexiguridad es más bien una pauta para el intercambio entre actores sociales, dentro de unas reglas de juego determinadas por las condiciones de los contextos en las que éstos interactúan. Así el posible intercambio se ciñe a la combinación entre diferentes políticas de flexibilidad y seguridad, como son las medidas de flexibilidad en el mercado de trabajo, las políticas activas de empleo (en particular las de recualificación y formación profesional) y las políticas de prestaciones por desempleo. La coordinación y el equilibrio entre estas tres políticas están sujetos a relaciones de negociación y conflicto. De ésta manera, el diálogo social se convierte en un mecanismo clave para encauzar el consenso de los actores sociales en torno a unas medidas de flexiguridad que resultan del intercambio entre actores con percepciones, poder de negociación y objetivos diferentes. Por consiguiente, el equilibrio entre flexibilidad y seguridad está vinculado a las contingencias propias de los sistemas 
de relaciones laborales y los mercados laborales nacionales. Así como a los valores e imaginarios colectivos que orientan y legitiman las actuaciones de los actores. De ello se deriva una posible explicación de la flexiguridad en términos de "efecto societal" (Maurice; Sellier; Silvestre, 1987) en el sentido de que el contexto histórico, el marco institucional, los actores sociales, la estructura económica y el ordenamiento jurídico de cada país, son clave para comprender la geometría variable de la flexiguridad, así como la diversidad de significados atribuidos a este controvertido concepto.

Debido a las limitaciones de espacio, no podemos en éste artículo entrar en el análisis comparativo detallado del contexto institucional dentro del cual entender e interpretar el desarrollo de las políticas de flexiguridad en Italia y España. El análisis siguiendo la estrategia de casos más similares nos permite, a partir de una breve descripción inicial de las similitudes institucionales entre los dos países, pasar a enfatizar las diferencias que ayuden a explicar resultados dispares en las políticas e implementación de flexiseguridad. Esto se hará en las siguientes secciones en las que se realiza el análisis de las diferencias en las tres dimensiones de flexiguridad.

En este sentido, y a diferencia de los países referentes del ideal de flexicuridad, en España e Italia abunda la pequeña empresa, que opera fundamentalmente en mercados locales y en espacios económicos con gran contenido informal. Los mercados de trabajo han sido estructurados en torno a la protección a la figura del male breadwinner, lo que repercute en un sistema de protección social que se encuentra fragmentado siguiendo una lógica ocupacional y que, en muchos casos, provee unos bajos niveles de cobertura. Además, en el Sur de Europa no se ha creado suficiente empleo de calidad, porque tampoco se ha logrado mejorar la capacidad competitiva en base a I+D (Investigación y Desarrollo), tal como se proponía en la Cumbre de Lisboa. El propio estado de bienestar es más débil y tardío, de carácter familista, basado en una fuerte división sexual del trabajo y una baja provisión de cuidado socialmente organizado (Bettio et alii., 2006) que tiene como resultado una combinación entre provisión pública de prestaciones y servicios que ha sido definido como Modelo de Bienestar Mediterráneo, contrapuesto precisamente al modelo escandinavo, tal como lo definió Esping-Andersen (2000), entre otros. Por tanto es cuanto menos arriesgado hablar de la flexiguridad como una alternativa para estos países cuando, en los últimos años, su crecimiento económico se ha basado en la expansión de un sector de servicios, con bajos salarios y bajos niveles de contribución fiscal, a parte del sector de la construcción, en el caso español. El escenario en el sur de Europa apunta, más bien, hacia el impulso de una política orientada hacia un nuevo "modelo social de bajo coste", que haga compatible la realidad de la flexibilidad del mercado laboral con la provisión de prestaciones y de servicios, en particular de servicios de cuidado de las personas, mínimos y baratos (Martín Artiles, 2008; Simonazzi, 2007).

De hecho, a pesar del énfasis de la Comisión Europea, los resultados hasta el momento parecen indicar que el paradigma de la flexiguridad no es fácilmente exportable (transponible) a otros países, dadas las diferencias significativos tanto en los ámbitos productivos y de trabajo, como en las instituciones y modelos de bien- 
estar. En cierto modo, así se reconoce desde voces autorizadas de la UE (Spidla 2005).

Debido a que los dos países han sido presentados tradicionalmente como pertenecientes a un modelo mediterráneo o del Sur de Europa, en la literatura comparada han tendido a enfatizarse las similitudes institucionales y en sus políticas. Sin embargo, a pesar de los muchos aspectos comunes entre los dos países, es importante tener presentes también algunas diferencias institucionales, aparte de las políticas que se mencionarán con posterioridad en el artículo. En primer lugar, existen disparidades institucionales relevantes en lo que se refiere a los marcos de relaciones laborales, y en particular, la presencia de sindicatos a nivel de empresa, los niveles de afiliación o la extensión de convenios. Asimismo, también existen diferencias significativas en lo referido al diálogo social, donde los dos países han seguido dinámicas claramente diferenciadas en los últimos quince años (Molina 2005). Pese a esto, la reciente crisis económica ha puesto de manifiesto la alta dependencia de las políticas de flexiguridad, no sólo en el contexto político como veremos a través de la comparación entre España e Italia, sino también en el contexto económico.

Teniendo en cuenta éstas diferencias y similitudes, exploramos la flexiguridad en España e Italia a través de las tres dimensiones o políticas que se consideran clave en dicho concepto, con objeto de facilitar el análisis comparativo. Esto es, la dimensión de la flexibilidad o rigidez del mercado laboral, la de las prestaciones por desempleo y la de las políticas activas de empleo, en particular, de aquellas ligadas a la formación. Para ello, utilizamos y contrastamos algunos de los indicadores más habituales en este tipo de análisis, junto a otros que han sido menos utilizados en los estudios realizados hasta la fecha ${ }^{2}$. Lo que nos ha permitido afinar en las similitudes y en las diferencias entre ambos países, así como entre Italia y España y el resto de países de la Unión Europea. De cara a simplificar el análisis, hemos seleccionado sólo algunos países, ilustrativos de los distintos modelos de empleo y de bienestar europeos, además de los casos danés y holandés considerados como modelo de flexiguridad.

\section{Mercado de trabajo flexible}

La primera cuestión a señalar es que no resulta fácil definir qué es un mercado laboral rígido o flexible, y mucho menos medirlo. Uno de los indicadores más utilizados es el Employment Protection Legislation Index (Indice de la Protección Legal del Empleo) de la OCDE, que intenta sintetizar el grado de flexibilidad o de rigidez de un mercado laboral. Según este indicador España mostraría unos niveles

${ }^{2}$ Los datos usados se han extraído de la base de datos Dataset for Flexicurity Analysis de la Red de Excelencia Europea RECWOWE (Reconciling Work and Welfare in Europe, (http://www.edacwowe.eu) 
de rigidez mayores que Italia, tanto para la mano de obra con contrato indefinido, como para los trabajadores y trabajadoras por cuenta ajena con contrato temporal (Tabla 1). Aunque cabe remarcar la existencia de tendencias opuestas con respecto a este último colectivo, puesto que en Italia se ha relajado de forma significativa su protección formal, pasando de ser 5,38 en 1990 a 2 en 2008 mientras que en España también ha disminuido aunque de forma leve desde 3,75 en 1990 hasta 3,5 en 2008 y ha mostrado una tendencia creciente en los últimos años a través de reformas negociadas con los actores sociales ${ }^{3}$. A pesar de ello y como resultado de las últimas reformas en los mercados de trabajo, el nivel de rigidez formal en el mercado de trabajo de trabajo italiano sería hoy día menor que en el español, lo cual acercaría más a Italia al ideal de mayor nivel de flexibilidad en el mercado laboral perseguido desde el paradigma de flexiguridad. Siempre teniendo en cuenta que los valores para Italia siguen siendo significativamente superiores a los de Dinamarca o, en menor medida, de Holanda.

Tabla 1

\begin{tabular}{|l|c|c|c|c|}
\hline \multicolumn{5}{|c|}{$\begin{array}{c}\text { Indicadores Objetivos de Protección al Empleo o Rigidez } \\
\text { Formal del Mercado de Trabajo }\end{array}$} \\
\hline & 1990 & 1998 & 2003 & 2008 \\
\hline España & 3,82 & 2,93 & 2,98 & 2,98 \\
\hline -Empleo Típico & 3,88 & 2,61 & 2,46 & 2,46 \\
\hline -Empleo Temporal & 3,75 & 3,25 & 3,5 & 3,5 \\
\hline Italia & 3,57 & 2,7 & 1,82 & 1,89 \\
\hline -Empleo Típico & 1,77 & 1,77 & 1,77 & 1,77 \\
\hline -Empleo Temporal & 5,38 & 3,63 & 1,88 & 2 \\
\hline Dinamarca & 2,4 & 1,5 & 1,5 & 1,5 \\
\hline Holanda & 2,73 & 2,73 & 2,12 & 1,95 \\
\hline France & 2,98 & 2,98 & 3,05 & 3,05 \\
\hline Alemania & 3,17 & 2,34 & 2,09 & 2,12 \\
\hline Suecia & 3,49 & 2,24 & 2,24 & 1,87 \\
\hline Reino Unido & 0,6 & 0,6 & 0,75 & 0,75 \\
\hline
\end{tabular}

${ }^{3}$ Es el caso del Acuerdo para la Estabilidad en el Empleo (AIEE) de 1997, y del Acuerdo Interconfederal para la Mejora de la Competitividad y el Empleo (AIMCE) de-2006, cuyas principales medidas contemplan subvenciones del Estado a las cuotas de la seguridad social de las empresas por la transformación de contratos temporales en estables, así como otras que reforman los contenidos de la negociación colectiva en materia de garantías, lo que se expresa en los convenios colectivos mediante cláusulas de limitación de la subcontratación, del empleo temporal, etc. 
Fuente: OCDE (2004).

(*) El indicador corresponde al Employment Protection Legislation Index versión 1 usado por la OCDE que sólo considera la regulación legal.

Basándose en los datos anteriores, se ha argumentado una alta rigidez en el mercado de trabajo español como causa de las altas tasas de desempleo que caracterizan la economía española (Scarpetta 1996). El mismo argumento, aunque en menor medida, ha sido también aplicado en el caso italiano. Al mismo tiempo, la alta protección de que goza el empleo por cuenta ajena en España se considera uno de los motivos de la existencia de un alto nivel de segmentación en los mercados de trabajo, fenómeno que también se observa en el caso de Italia. Sin embargo, las diferencias entre el caso español e italiano que arroja el indicador OCDE deben ser contrastadas con otros indicadores. Así pues, usando datos de la Encuesta de Mercado de Trabajo (Labour Force Survey) y del Panel de Hogares de la Comunidad Europea (European Community Household Panel), puede apreciarse como la movilidad horizontal en el mercado de trabajo español es significativamente mayor que la italiana o la de cualquier país europeo. Esto es en gran parte debido a una mayor rotación en el empleo por causa de la mayor temporalidad (Tabla 2). Estos resultados son confirmados en el reciente estudio de la Fundación Europea para la Mejora de las Condiciones de Vida y Trabajo (European Foundation for the Improvement of Working and Living Conditions) (2008: 18-19) que muestra una mayor movilidad en el caso de España en comparación con Italia, pero similares niveles de seguridad dinámica en el empleo. Por tanto, al menos en el caso español, el indicador que más comúnmente se usa para valorar la flexibilidad del mercado de trabajo, el de la OCDE, arroja una visión sesgada ya que no refleja todas las dimensiones de la flexibilidad relevantes para valorar el funcionamiento agregado del mercado.

Tabla 2. Tasa de Movilidad Horizontal en el Mercado de Trabajo: Ingresos y Salidas del Status Empleado

\begin{tabular}{|l|l|l|}
\hline & LFS & ECHP \\
\hline España & 29,2 & 32,2 \\
\hline Italia & 23,6 & 22,4 \\
\hline Dinamarca & 22,6 & 18,7 \\
\hline Holanda & 18,7 & 22,5 \\
\hline Francia & 20,2 & 12,3 \\
\hline Alemania & 16,3 & 16,1 \\
\hline Suecia & nd & nd \\
\hline Reino Unido & 19,7 & 21 \\
\hline
\end{tabular}

Fuente: Kruppe (2001, tabla 2.5). La tabla recoge indicadores de tasa de movilidad en el mercado de trabajo 
según dos fuentes: LFS (Labour Force Survey) y ECHP

(European Community Household Pannel).

La imagen que arrojan los principales indicadores OCDE, así como la interpretación que de éstos se ha hecho sobre todo por economistas, es que los mercados de trabajo en España e Italia son comparativamente más rígidos que los del resto de países europeos debido a la excesiva protección normativa que se provee a los empleados por cuenta ajena, sobre todo a aquellos con un contrato estable a tiempo indeterminado. Sin embargo ésta imagen no se corresponde con la percepción subjetiva de seguridad en el empleo, tal como muestran los datos de la Encuesta Europea de Calidad de Vida (European Quality of Life Survey) 2007, donde se observa como la percepción de inseguridad es significativamente mayor en España que en Italia u otros países europeos. Una aparente contradicción que se explica, en buena medida, por un factor estructural: el patrón de industrialización y de desarrollo económico de las economías mediterráneas favorece unos mercados laborales generadores de un empleo débil tanto por la alta segmentación y segregación que los caracteriza, como por las altas tasas de desempleo y las condiciones laborales medias, en particular las referidas a la contratación temporal, en el caso español. Todos estos aspectos escapan al índice OCDE de protección al empleo y por tanto este indicador ofrece una visión distorsionada de la realidad de los mercados laborales.

En lo que se refiere a las diferencias entre los dos países mediterráneos, destaca la disparidad de trayectorias que han seguido España e Italia desde principios de los años 80. La introducción de flexibilidad en España se produjo a partir de mitad de los años 80 con la reforma laboral de 1984 que eliminó el principio de causalidad en la contratación temporal. A partir de ésta reforma, se produjo un crecimiento rápido en este tipo de contratos y pese a algunos intentos recientes con el objetivo de reducir la temporalidad, ésta se mantiene alrededor del $30 \%$ de la población ocupada. La evolución en Italia ha sido diferente. Los años 80 se caracterizaron por el mantenimiento de la regulación heredada de los años 70 . La fuente de flexibilidad numérica en el mercado de trabajo italiano ha sido tradicionalmente la existencia de un gran número de trabajadores autónomos o falsos autónomos que mantenían relaciones estables con empresas. Sólo a partir de la segunda mitad de los años 90 se han producido intentos de flexibilizar el mercado de trabajo, extendiendo el uso de formas atípicas de empleo. Así pues, pese a que ha habido una cierta divergencia en el corto plazo más reciente, la trayectoria de largo plazo parece apuntar hacia una cierta convergencia.

\section{Prestaciones por desempleo}

Uno de los argumentos más recurrentes en el debate de la flexiguridad pone de relieve las bondades del modelo danés, que ofrece unas importantes prestaciones por desempleo a cambio de facilidades en el despido. Esto contrasta con la práctica en los países del sur de Europa, donde existe una alta protección del empleo (i.e., dificultades para despedir) y una escasa generosidad en sus prestaciones por desem- 
pleo. Un estudio de Boeri y otros (2006) compara el "índice de rigidez del mercado de trabajo" y el "índice de generosidad de la prestación por desempleo" ${ }^{5} \mathrm{de}$ los países de la UE. El estudio muestra como los países nórdicos (Dinamarca, Finlandia, Bélgica y Suecia) tienen mercados laborales flexibles, que facilitan la movilidad de la fuerza de trabajo, al mismo tiempo que ofrecen una alta protección por desempleo, mientras que, por el contrario, los países del sur (Grecia, Italia, Portugal y España), tienen una alta protección del mercado de trabajo y bajas prestaciones por desempleo. De esta forma Boeri confirma que los países que constituyen un modelo de flexiguridad son aquellos que consiguen combinar una gran flexibilidad para las empresas con un alto grado de seguridad para los individuos en forma de altas prestaciones por desempleo.

Cuando miramos a los programas de mantenimiento de rentas para desocupados, es necesario distinguir entre su generosidad (porcentaje de sustitución de la renta salarial) y duración (periodo de percepción). La relación entre éstas dos dimensiones queda reflejada en el gráfico 2 para diversos países europeos. En general, puede hablarse de una relación positiva entre duración y generosidad. Sin embargo cabe destacar el caso de Dinamarca, donde a igual nivel de generosidad que otros países, su duración alcanza los 48 meses cubriendo aproximadamente el $70 \%$ del salario, aunque puede llegar también el $90 \%$ del salario según los casos y la situación familiar. En el caso español la duración máxima del seguro de desempleo son 24 meses y cubre entre el 60 y $70 \%$ del salario. Es decir, aparentemente el español es más generoso que el sistema italiano, que sólo cubre 8 meses y la cuantía de la pensión apenas representa algo más del $60 \%$ del salario anteriormente percibido para los primeros seis meses, para después pasar al 50\% (ETUI, 2008).

${ }^{4}$ Se define el "Índice de rigidez" (acrónimo en inglés, EPL) como el coste burocrático y del procedimiento legal de la protección del seguro desempleo (Boeri, 2006:10-11).

${ }^{5} \mathrm{El}$ "Índice de generosidad de la prestación por desempleo" (acrónimo en ingles: UB) se ha construido a partir del total de recursos destinados al desempleo y su distribución entre los desempleados en cada país (Boeri, 2006:9-10). 
Gráfico 2. Relación entre la duración del beneficio por desempleo y la tasa de sustitución salarial en Europa 2005

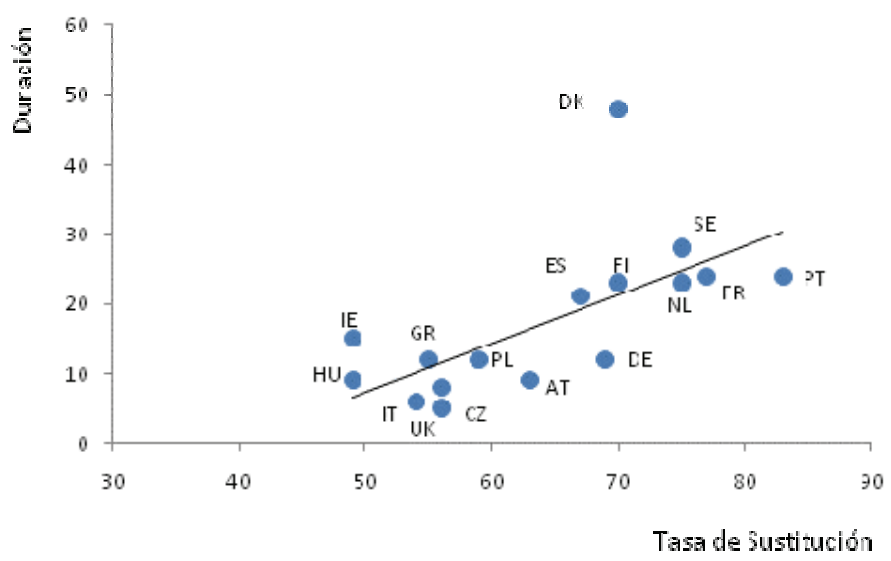

Fuente: OCDE, 2006.

Si lo comparamos con otros países europeos, vemos como en el Reino Unido se ofrece sólo una cobertura mínima, modelada según la edad de la persona en situación de desempleo. En concreto, sólo tiene derecho a un máximo de 66,08 euros semanales si se trata de una persona mayor de 25 años, de 52,34 euros si tiene entre 18 y 24 años, y de 40,61 euros para los jóvenes de 16 y 17 años. A cambio, hay que demostrar que se está buscando activamente empleo, como en España, pero el tiempo de la prestación es mucho más reducido. En este sentido, el caso británico es un ejemplo de lo que persigue la política de "empleabilidad" que impulsa la UE: disponibilidad y búsqueda activa para la reinserción inmediata al mercado laboral. En otros países dentro del modelo continental como son Alemania y Francia, las pensiones ofrecen más garantías en la percepción de rentas. Así, en Alemania existe por un lado una pensión contributiva que se percibe en los 12 meses que siguen a la pérdida del empleo, y que cubre entre el $60 \%$ y el $67 \%$ del salario. El segundo tipo es una pensión complementaria que sustituye a la anterior cuando se agota el periodo de derecho a percepción, o la complementa. Por su parte, en Francia, el seguro de desempleo tiene una duración de dos años. Cuando se agota éste periodo, puede accederse a la llamada Prestación Solidaria Específica, que tiene una duración de seis meses renovables sin límite de tiempo, y cuya cuantía máxima es de 450 euros mensuales. Es un mecanismo parecido al recientemente implantado en España, consistente en un subsidio complementario de 420 euros durante 6 meses, para 
aquellas personas que hayan agotado el derecho a pensión contributiva por desempleo ${ }^{6}$.

En este sentido, podría concluirse que uno de los aspectos diferenciales entre España e Italia es precisamente la diferencia en la generosidad y cobertura del subsidio de desempleo. A juzgar por los datos agregados de la OCDE, tanto el nivel de generosidad (medida como la tasa neta de sustitución del subsidio con respecto al salario medio de los meses anteriores) como la duración (el número de meses durante los cuales un individuo puede obtener el subsidio) son mayores en el caso español. Por otro lado, las condiciones de acceso al subsidio medido en número de días trabajados en el período inmediatamente anterior al desempleo son también significativamente más restrictivas en el caso italiano (ver tabla 4). Además este último país presenta una mayor selectividad y fragmentación de las prestaciones de indemnización por categorías de trabajadores con base en el sector de pertenencia, las dimensiones de empresa, el tipo de contrato, la edad y por área geográfica, como ampliaremos más adelante.

Tabla 4. Pensiones por desempleo para beneficiarios mayores de 40 años y con 22 años de empleo contributivo a la seguridad social

\begin{tabular}{|c|c|c|c|}
\hline & Italia & España & Dinamarca \\
\hline $\begin{array}{l}\text { Empleo y condiciones } \\
\text { de contribución }\end{array}$ & $\begin{array}{l}52 \text { semanas en dos } \\
\text { años }\end{array}$ & $\begin{array}{l}52 \text { semanas en seis } \\
\text { años }\end{array}$ & $\begin{array}{c}52 \text { semanas en tres } \\
\text { años }\end{array}$ \\
\hline $\begin{array}{l}\text { Duración máxima de la } \\
\text { prestación (meses) }\end{array}$ & 7 & 24 & 48 \\
\hline $\begin{array}{l}\text { Paga inicial (En \% } \\
\text { sobre la base del salario } \\
\text { percibido) }\end{array}$ & $\begin{array}{c}50 \% \\
\text { (40\% después de } \\
\text { seis meses) }\end{array}$ & $\begin{array}{c}70 \% \\
\text { (60\% después de } 6 \\
\text { meses) }\end{array}$ & $90 \%$ \\
\hline $\begin{array}{l}\text { Base de la pensión por } \\
\text { desempleo }\end{array}$ & $\begin{array}{c}\text { Media sobre el } \\
\text { salario bruto en los } \\
\text { tres últimos meses } \\
\text { previos al desempleo }\end{array}$ & $\begin{array}{l}\text { Media del salario } \\
\text { bruto }\end{array}$ & $\begin{array}{l}\text { Salario bruto del } \\
\text { último empleo } \\
\text { menos } 8 \% \text { de } \\
\text { contribuciones a la } \\
\text { seguridad social }\end{array}$ \\
\hline $\begin{array}{l}\text { Percepción mínima } \\
\text { pensión }\end{array}$ & -- & 4.510 euros & -- \\
\hline $\begin{array}{l}\text { \% sobre la } \\
\text { el Salario } \\
\text { Medio }\end{array}$ & -- & $22 \%$ & $43 \%$ \\
\hline
\end{tabular}

${ }^{6}$ Real Decreto Ley 10/2009 del 13 de agosto por el que se regula el programa temporal de protección por desempleo e inserción. 


\begin{tabular}{|c|c|c|c|}
\hline Percepción máxima & 11.821 euros & 9.866 euros & \\
\hline $\begin{array}{l}\% \text { sobre el } \\
\text { Salario } \\
\text { Medio }\end{array}$ & $52 \%$ & $48 \%$ & $53 \%$ \\
\hline $\begin{array}{l}\text { Permiso para realizar o } \\
\text { desempeñar trabajos } \\
\text { ocasionales }\end{array}$ & $\begin{array}{l}\text { No hay derecho a } \\
\text { beneficio si se } \\
\text { trabaja, excepto si se } \\
\text { está cobrando CIG }\end{array}$ & $\begin{array}{l}\text { Reducción de la } \\
\text { pensión en función } \\
\text { de las horas trabaja- } \\
\text { das. }\end{array}$ & $\begin{array}{l}\text { El salario percibido } \\
\text { se reduce totalmente } \\
\text { del beneficio }\end{array}$ \\
\hline \begin{tabular}{|l|} 
Complementos por \\
familiares dependientes
\end{tabular} & -- & $\begin{array}{l}\text { Incremento de la } \\
\text { pensión por tener } \\
\text { hijos a cargo. }\end{array}$ & \\
\hline
\end{tabular}

Fuente: OECD, Unemployment Benefits Database.

Las tasas de sustitución de los salarios en los meses previos al desempleo, son prácticamente iguales en los dos países para los meses iniciales. Aunque existen algunas diferencias dependiendo de la situación familiar ya que, en el caso de España, para un nivel salarial en torno a la media (100\% media salarial), el grado de sustitución es inferior comparado con Italia para desempleados sin familiares dependientes a cargo, mientras que es mayor cuando existen cargas familiares. Sin embargo, a medida que se alarga el periodo, el grado de sustitución de renta salarial en España es significativamente mayor para todos los niveles salariales y estados familiares. Es decir, el sistema español se muestra más generoso que el sistema italiano y genera una mayor seguridad a los trabajadores que se enfrentan a periodos largos de desempleo independientemente de su situación familiar (gráfico 3). Como veremos a continuación, ésta es la principal razón para explicar el mayor gasto en políticas pasivas del mercado de trabajo en España comparado con Italia. Por otra parte, las diferencias en niveles y duración de la prestación entre España e Italia también encuentran reflejo en los datos subjetivos sobre la (des) confianza en la percepción de asistencia a través de programas de mantenimiento de rentas, donde podemos observar un mayor nivel de confianza en el caso español, según datos del European Quality of Life Survey 2003. Quizá porque la mayor tasa de empleo femenino en España sugiere que existe un mayor porcentaje de familias que encuentran protección en sus rentas (seguridad) de forma privada, i.e., a través de unos segundos ingresos en el mercado de trabajo. 
Gráfico 3 Tasa de sustitución (\% salario bruto) de la prestación de desempleo. España e Italia 2005

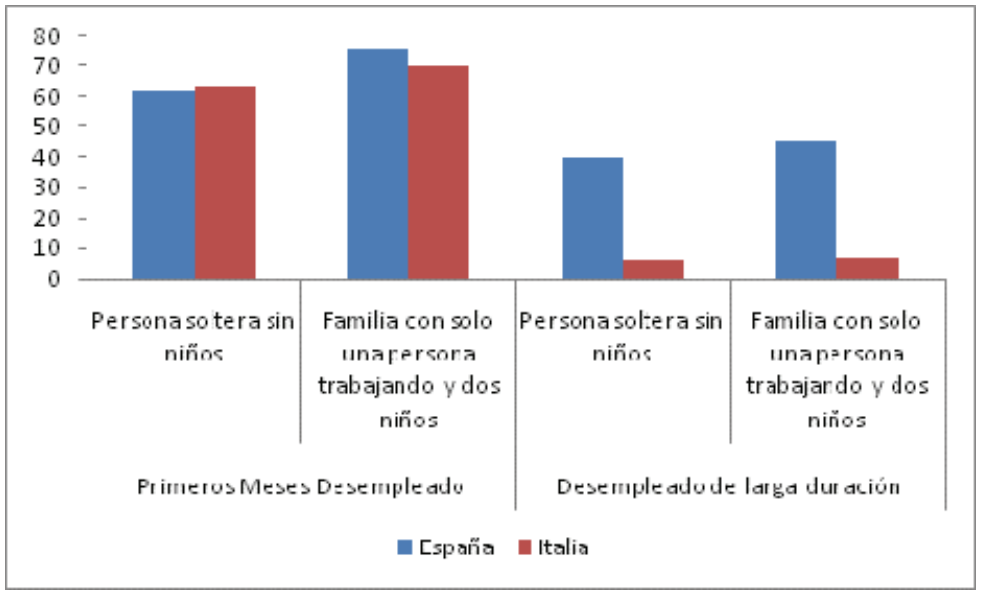

Fuente: OECD, Unemployment Benefits Database

A pesar de la aparente rotundidad de estos datos, que no dejarían lugar a dudas a la hora concluir la mayor generosidad del subsidio para el caso de España, conviene hacer una serie de puntualizaciones para no incurrir en conclusiones apresuradas y erróneas. Para ello es necesario adoptar una visión más general del mercado de trabajo, así como mirar más en profundidad los sistemas de sustitución de rentas para las personas en paro y, al mismo tiempo, analizar las últimas reformas en estos sistemas para poder realizar un diagnóstico más completo de la situación en estos países con respecto al subsidio de desempleo.

En efecto, un primer aspecto a destacar en el caso de España es el alto nivel de empleo temporal, compuesto en su mayoría por contratos de duración menor a un año y con bajos salarios. Ello significa que un importante colectivo de trabajadores y de trabajadoras encuentra serias dificultades para poder acceder a una pensión contributiva por desempleo, puesto que no llegan a trabajar el suficiente número de semanas exigido por la ley, y en caso de acceder, lo hacen con una prestación de poca cuantía debido a los bajos salarios que han servido de base de cotización. Por lo tanto, los altos niveles de temporalidad en España no sólo implican que la flexibilidad e inseguridad en el mercado de trabajo son mayores de lo que pueden mostrar los indicadores agregados sintéticos (debido a que la contratación temporal apenas representa un coste por finalización de contrato), sino que la cobertura de la pensión por desempleo es también menor de lo que podría creerse a priori. Esto es especialmente significativo para aquellos colectivos más expuestos a formas débiles de relación con el empleo.

Con todo, las prestaciones por desempleo han mejorado a través de la Ley 43/2006, que recoge a su vez las recomendaciones de las Directivas de la Estrategia 
Europea de empleo y del Acuerdo para la Mejora de la Competitividad y el Empleo (AMCE) suscrito por los agentes sociales en el año 2006, en España. La ley introduce mejoras en las prestaciones por desempleo para determinados colectivos, como son el personal fijo discontinuo, los mayores de 45 años, los trabajadores socios de cooperativas, algunos colectivos de trabajadores agrarios, así como a determinados cargos políticos y sindicales - en este último caso, de acuerdo con la Ley 37/2006, de 7 de diciembre. A esta provisión de seguridad cabe añadir la del Estatuto de los Trabajadores Autónomos, que ha sido considerada como un ejemplo de las políticas públicas ejemplares.

Gráfico 4. Evolución en el Indicador Sintético $(*)$ de Derecho a la Percepción de subsidio por desempleo. Italia y España.

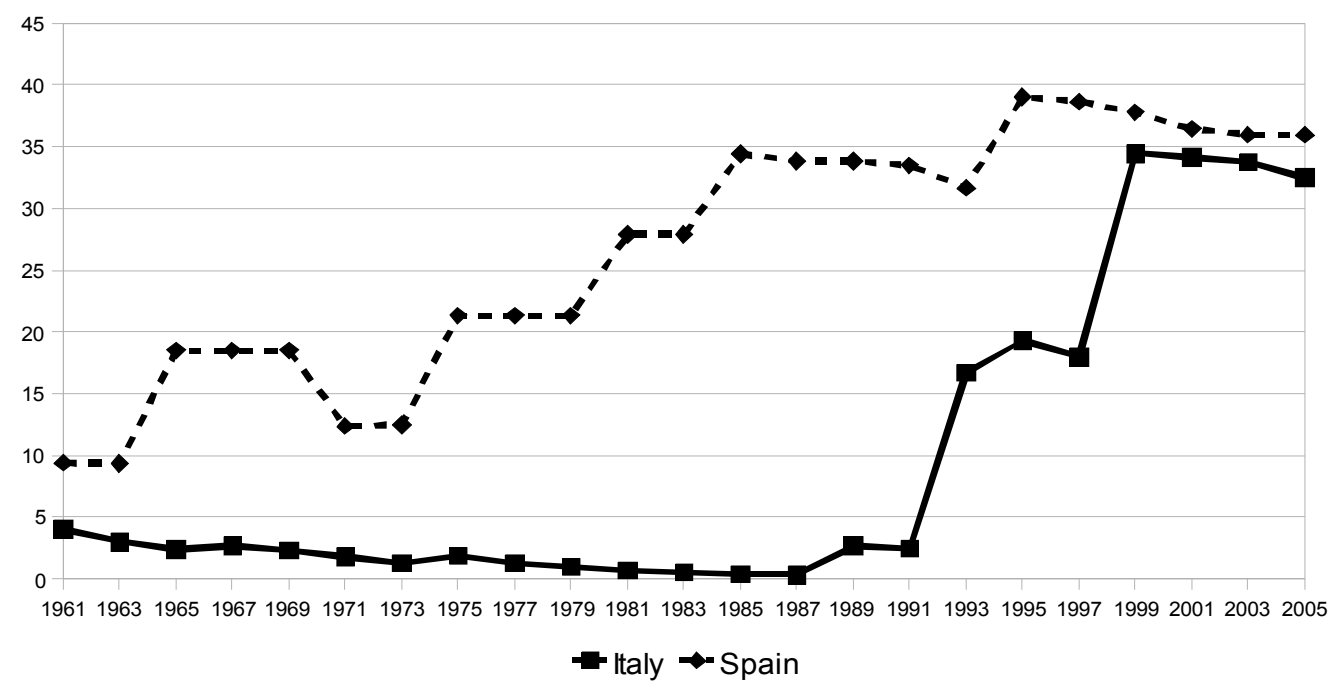

(*) Dicho indicador se define como la media de la tasa de sustitución del subsidio de desempleo para dos niveles de desempleo, tres situaciones familiares y tres duraciones del status de desempleado. Ver OECD (1994), The OECD Jobs Study (capítulo 8) y J. Martin (1996) "Measures of Replacement Rates for the Purpose of International Comparisons: A Note", OECD Economic Studies, No. 26. Los datos anteriores a 2003 han sido revisados.

Sin embargo, cuando comparamos los casos italiano y español, yendo más allá de ese tipo de indicadores, existen dos aspectos a destacar. En primer lugar, la fragmentación en el sistema de pensiones italiano dificulta la valoración objetiva del grado de generosidad y cobertura de las prestaciones por desempleo. De hecho, como detallamos a continuación, existen otros programas que contribuyen a mejorar el nivel de prestaciones que provee el sistema contributivo ordinario de subsidio de desempleo. En segundo lugar, mirando a las recientes reformas en el sistema italiano puede observarse una tendencia hacia la extensión del subsidio, tanto en el grado 
de sustitución del salario como en la duración de la prestación. Lo que ha provocado una cierta convergencia entre ambos países (gráfico 4).

La fragmentación del sistema italiano de pensiones por desempleo es una de sus características más sobresalientes. Existe, en primer lugar, el sistema contributivo de pensiones por desempleo (Indennità di Disoccupazione) que históricamente se ha caracterizado por tener unas tasas muy bajas de sustitución y poca cobertura. En segundo lugar se encuentran los esquemas industriales de la Cassa Integrazione Guadagni (CIG), o fondo de compensación salarial acordado a través de la negociación colectiva. A través de este fondo, los trabajadores de empresas medianas y grandes en el sector industrial que se encuentran en momentos cíclicos de baja producción y que como consecuencia sufren una merma en sus ingresos por una reducción de su jornada, pueden acceder a una compensación por el resto de horas que dejan de trabajar. Este sistema ha actuado como verdadera protección al desempleo con un nivel de generosidad alto comparado con el sistema contributivo. Sin embargo, este sistema sólo ha provisto cobertura a un grupo hoy reducido de trabajadores como son los blue-collar de empresas medianas y grandes. Y, en tercer lugar, la Cassa Integrazione Guadagni Straordinaria que no sólo compensa los efectos de una reducción cíclica o temporal, sino que se aplica también a casos de re-estructuración industrial, es decir, de cierre de empresa.

Todo ello no impide que, en Italia, estén sin cobertura la totalidad de los trabajadores "parasubordinati", o "falsos autónomos", y del 38\% al 79\% de los trabajadores dependientes con contratos a duración determinada, pero también más del $10 \%$ de los trabajadores a tiempo indeterminado. Se estima que 1,6 millones de trabajadores dependientes y "parasubordinati" no tienen derecho a ningún sostén en caso de despido (Banco de Italia 2009: 12) y que al menos el doble a los trabajadores están excluidos de las indemnizaciones de paro, las prestaciones ordinarias e incluso de lo que prevén los requisitos reducidos o mínimos (Berton, Richardi y Sacchi, 2009). Por último, cabe señalar que, ante la última crisis, el gobierno Berlusconi ha adoptado medidas que conceden indemnizaciones de paro y sostén de las rentas (a través de la CIG) cuando se agotan las prestaciones por desempleo. Las acciones tomadas han apuntado en dos direcciones. Por un lado, se ha extendido la CIG para abarcar a empresas medianas y pequeñas, lo que ha supuesto la re-valorización de un esquema contributivo que había sido relegado a un papel marginal. Por otro lado, el reciente decreto italiano anticrisis ${ }^{7}$ ha fomentado una variedad de acuerdos entre las regiones y los agentes sociales para la concesión de la indemnizaciones por movilidad, finalización de contrato, así como también a trabajadores empleados con formas contractuales atípicas, aunque el desarrollo de estas medidas difiere de una región a otra. En suma, en el caso italiano, se observa una tendencia a intervenir sin

${ }^{7}$ Decreto Ley n. 185 de 2008 convertido en Ley con modificación de la Ley n. 2 de 2009 y modificado posterioremente con la ley n 33 del año 2009. 
una lógica universalista a través de acciones a nivel regional que alimentan ulteriores disparidades de trato entre categorías de trabajadores y trabajadoras.

\section{Políticas activas de empleo}

Siguiendo con el esquema ideal-típico de flexiguridad, el segundo mecanismo que junto con las pensiones por desempleo debe acompañar la mayor flexibilidad en el mercado laboral son las políticas activas, y más específicamente, las de fomento del empleo, re-cualificación y formación. Las políticas activas de empleo se han venido desarrollando en base a dos grandes líneas: 1) las políticas de fomento y gestión del empleo y 2) las relativas a los programas de formación en general. Entre las primeras se encuentran las políticas destinadas a mejorar la conexión entre la oferta y la demanda de empleo, por ejemplo a través de la mejora de los servicios públicos de empleo, los planes de contratación para desempleados, los incentivos a la contratación de discapacitados, el apoyo al autoempleo, así como otros programas activos de dinamización y movilidad del colectivo de trabajadores desempleados. Dentro de las segundas se incluyen todas las políticas de formación continua y re-cualificación de trabajadores en paro.

La creciente importancia de éste tipo de políticas queda plasmada en el aumento del gasto en España en los últimos años. Sin embargo, existe una brecha muy significativa entre el gasto en políticas pasivas (superior a la media UE) y el gasto en política activas (inferior a la media UE). Entrando en el detalle del gasto en políticas activas, un aspecto distintivo del caso español es que el gasto en incentivos directos al empleo, a través de bonificaciones a las empresas, es superior a la media de la UE-15 (14,6\% frente a 6,4\%, según CES, 2008:363), lo que puede entenderse también como una fórmula para reducir costes indirectos del trabajo y por tanto como una forma pasiva de aumento de la competitividad de las empresas. Pero, a la vez, refleja los intentos gubernamentales para transformar el empleo temporal en empleo estable con la finalidad de mejorar la formación, las cualificaciones y la competitividad, tal como se refleja en el Acuerdo Interconfederal para la Mejora de la Competitividad y el Empleo (AIMCE 2006).

Según la misma fuente, el gasto en servicios a la intermediación entre la oferta y la demanda en el mercado laboral es también menor que la media europea. No obstante, éste ha aumentado en los últimos años ya que uno de los objetivos que más insistentemente se ha venido desarrollando en el marco de las políticas activas, es la modernización y la mejora de los servicios de mediación entre la oferta y la demanda en el mercado de trabajo, el tratamiento informático de la información y la identificación del colectivo de desempleados para definir los distintos grados de empleabilidad. En el mismo sentido, también el gasto en formación es inferior a la media de la UE-15, siendo éste precisamente un objetivo prioritario de las políticas de la UE para mejorar la competitividad y la empleabilidad en el marco de la sociedad del conocimiento. 
Comparado con Italia, el gasto en políticas de empleo en España es superior como porcentaje del PIB. El gasto en políticas activas de empleo por punto de la tasa de paro es superior, como también es superior el gasto total en políticas de empleo (Tabla 5). No obstante, en ambos países el gasto es inferior a la media UE-15. La principal diferencia entre España e Italia viene marcada por el mayor gasto en políticas pasivas en el primero, y en particular por los programas de sostenimientos de rentas por desempleo más generosos y de mayor duración en España. Una diferencia notablemente menor en el caso de las políticas activas.

Tabla 5. Gasto en Políticas de Empleo en la UE-15 (en \% PIB 2006)

\begin{tabular}{|l|r|r|r|}
\hline & España & \multicolumn{1}{l|}{ Italia } & UE-15 \\
\hline Servicios mercado de trabajo & 0,1 & 0 & 0,2 \\
\hline Políticas activas (2-7) & 0,6 & 0,4 & 0,5 \\
\hline Políticas pasivas (8-9) & 1,4 & 0,8 & 1,3 \\
\hline Gasto total & 2,2 & 1,3 & 2 \\
\hline $\begin{array}{l}\text { Gasto total políticas activas por punto de tasa de } \\
\text { paro }\end{array}$ & & & \\
\cline { 2 - 4 } & 0,074 & 0,066 & 0,069 \\
\hline Gasto total por punto tasa paro & 0,255 & 0,188 & 0,262 \\
\hline
\end{tabular}

Fuente: Eurostat, Labour Market Policy. Expenditure and participation

Data 2006, 2008. Citado en CES (2008:362)

Con respecto al gasto en políticas activas, tanto los datos OCDE como los datos de Eurostat (Tabla 6) arrojan una imagen parecida en la que ambos países muestran niveles comparativamente bajos y similares entre ellos. Los datos Eurostat referidos a 2005 muestran un mayor nivel de gasto para el caso de España comparado con el año 2003 que sirve de referencia para los datos OCDE. En cualquier caso, los niveles de los dos países están muy lejos de los que presentan otros países europeos. Para finalizar, si que puede observarse una mayor participación de la población adulta entre 25-64 española en programas de formación continua, lo que sugiere que el elemento pasivo de seguridad (subsidio de desempleo) es acompañado de medidas de activación para permitir una más rápida re-colocación o mejorar la versatilidad de la mano de obra. Todos los indicadores, tanto de porcentaje de trabajadores como de número de horas de formación realizadas, muestran que la población adulta en edad de trabajar realiza más formación en el caso español, aunque con distinto peso según los colectivos. Así, los datos muestran como en el caso italiano, el porcentaje de personas por encima de los 55 años sobre jóvenes (24-32) que realizan formación es mayor que en el caso de España, donde la formación está especialmente dirigida a los jóvenes (Eurostat). En ambos casos, las actividades de formación para adultos las llevan a cabo sobre todo los hombres tanto en Italia como en España, y éstas priorizan la formación general (general skills) por encima 
de la formación específica en el lugar de trabajo (workplace training) o de la formación a través de la institución del aprendizaje.

Tabla 6. Gasto en Políticas Activas y Pasivas (en \%PIB). 2005

\begin{tabular}{|l|c|c|c|}
\hline & $\begin{array}{c}\text { Gasto en Políti- } \\
\text { cas Activas }\end{array}$ & $\begin{array}{c}\text { Gasto en Políti- } \\
\text { cas Pasivas }\end{array}$ & $\begin{array}{c}\text { Gasto en Políticas Activas pon- } \\
\text { derado por tasa de desempleo }\end{array}$ \\
\hline España & 0,583 & 1,455 & 0,063 \\
\hline Italia & 0,461 & 0,816 & 0,060 \\
\hline Dinamarca & 1,433 & 2,514 & 0,299 \\
\hline Holanda & 0,852 & 2,021 & 0,181 \\
\hline Francia & 0,664 & 1,625 & 0,072 \\
\hline Alemania & 0,616 & 2,346 & 0,058 \\
\hline Suecia & 1,097 & 1,198 & 0,148 \\
\hline Reino Unido & 0,116 & 0,187 & 0,024 \\
\hline
\end{tabular}

Fuente: Eurostat

\section{Conclusiones}

Para concluir, en los siguientes párrafos intentaremos dar respuesta a los dos interrogantes principales que nos plateábamos al inicio de éste artículo. En primer lugar, hasta qué punto los patrones de flexibilidad y seguridad en España e Italia nos permiten hablar de un modelo mediterráneo como tal, o por el contrario las diferencias que existen entre ambos hacen que el uso del término sea problemático cuando nos movemos en la relación entre mercado de trabajo y política social. En segundo lugar, mirando a la evolución de las políticas relacionadas con las tres dimensiones básicas del concepto de flexiguridad, hasta qué punto la transposición del paradigma ideal de flexiguridad en países como España e Italia es viable y hay un acercamiento a las políticas y resultados a los que se observan en países como Dinamarca.

A parte de confirmar la distancia entre el paradigma ideal de flexiguridad danés y los países mediterráneos y las dificultades de países como España e Italia para implementar un equilibrio similar entre flexibilidad laboral y seguridad, nuestra primera pregunta tiene como objetivo ir más allá de la simple catalogación de Italia y España como países con mercados de trabajo rígidos y poca seguridad para los trabajadores en sus transiciones del empleo al desempleo. El análisis en profundidad de cada una de las dimensiones centrales de la flexiguridad para ambos países, ha mostrado la existencia de diferencias y divergencias significativas. Evidentemente, al tratarse de dos países con similares estructuras en sus mercados de trabajo, las diferencias entre los dos casos deben ponerse en el contexto europeo para evitar su sobre-dimensionamiento. Pero un análisis más detallado permite sacar a la luz diferencias que pueden resultar importantes pero que análisis más generales no 
llegan identificar. En este sentido, vale la pena destacar la distorsión que introducen algunos indicadores objetivos sintéticos de rigideces en el mercado de trabajo, como queda reflejado cuando se presta atención a la movilidad y la rotación en el mercado de trabajo. Así pues, pese a que el indicador OCDE apunta a una mayor rigidez del mercado de trabajo español, los datos sobre rotación muestran que esa afirmación es cuanto menos discutible.

Del mismo modo, existen diferencias significativas en cuanto a las características de los programas de mantenimiento de rentas para desempleados. Éstas diferencias se refieren tanto a la generosidad como a la duración de las prestaciones por desempleo y en ambos caso los indicadores muestran una mayor cobertura al desempleo en España. Al mismo tiempo, las prestaciones en el caso italiano están mucho más fragmentadas según diferentes programas, lo cual contrasta con el caso español, donde rige un principio más universalista. Finalmente, en el caso de las políticas activas de empleo, también han sido identificadas diferencias, aunque en este caso sin revestir la misma relevancia que en las dos anteriores dimensiones.

Aún más importante que las diferencias observadas a partir del análisis estático de las tres dimensiones, son las divergencias que se han dado en la evolución de las políticas a lo largo de las últimas tres décadas. Esto queda patente en el caso de la regulación del mercado de trabajo y la introducción de flexibilidad, como apuntamos en la sección 2. Al mismo tiempo, en el caso de las pensiones por desempleo, a pesar de la persistencia de una mayor fragmentación de programas, en el caso italiano se observa una tendencia a aumentar tanto su generosidad como duración.

¿Qué explica las diferencias observadas a corto plazo y hasta qué punto éstas nos ofrecen indicios acerca de la viabilidad de la transposición del paradigma de flexiguridad? Una primera variable que se muestra relevante en la comparación entre España e Italia es la política. En este terreno podríamos concluir que el color de los gobiernos sigue jugando un papel importante. En esta última etapa, la existencia de gobiernos pro-labour, como ha sido el partido socialista en España, o la prevalencia de gobiernos conservadores-liberales y de división sindical como ha sido el caso de Italia en el periodo estudiado, nos ayudan a explicar la divergencia en las políticas de empleo. En Italia tiende a desarrollarse más la contratación temporal, que se encamina hacia una menor protección de las garantías del status del empleo; lo que se explica por la tardía puesta en práctica de medidas de contratación flexible. Por el contrario, en España, las medidas de contratación flexible son mucho más antiguas, introducidas a mediados de los años 80 . Hoy, la orientación política dominante consiste en introducir medidas más garantistas, tales como la transformación de empleo temporal en estable y otras limitaciones de las contrataciones atípicas a través de cláusulas en la negociación colectiva.

Pasando a la segunda pregunta, hasta qué punto partiendo de la evidencia anterior podemos hablar de una convergencia entre España e Italia hacia un modelo de flexiguridad como el danés, lo que nos ayuda también a concluir acerca de la viabilidad de la implementación de la flexiguridad. En este sentido conviene distinguir entre tendencias de corto y largo plazo. Así pues, si miramos a corto plazo, podríamos concluir que existe una clara divergencia en las experiencias española e italiana 
en un aspecto clave como es la regulación de la flexibilidad del mercado de trabajo. Algo similar podría concluirse al analizar la evolución de las prestaciones por desempleo. Sin embargo, adoptando una visión más amplia, podría llegarse a concluir exactamente lo contrario, es decir que se observa una cierta convergencia entre ambos países en lo que respecta a la flexibilidad cuantitativa en el mercado de trabajo. No obstante, es difícil determinar en base a la evidencia presentada en este artículo si se trata de un efecto real que apunte a una convergencia efectiva en el largo plazo.

Lo anterior nos permite aventurar algún tipo de conclusión con respecto a la posibilidad de transponer o implementar el paradigma de la flexiguridad en España e Italia. Así pues, las experiencias española e italiana ofrecen una evidencia que no se ajusta a las predicciones de la visión neofuncionalista de convergencia desde arriba. La convergencia inducida por la Estrategia Europea de Empleo, que ha adoptado el paradigma de flexiguridad, no parece estar ocurriendo. En primer lugar, a pesar de que el método abierto de coordinación como mecanismo de gobernanza de la EEE, ya presupone la posibilidad de variación en cuanto a la implementación de una misma orientación, el factor más importante que sigue determinando diferencias son los contextos socio-productivos nacionales, el poder de los actores y las instituciones políticas.

La correlación de fuerzas entre actores sociales a nivel nacional sigue siendo una fuente de diversidad, tanto entre países que pertenecen a un mismo modelo, como entre modelos sociales. Así pues, y pese a la insistencia de la Comisión Europea, u otros organismos internacionales, existen fuertes resistencias a introducir mayor flexibilidad en los mercados de trabajo del sur de Europa porque ésta es quizás la única vía de proporcionar seguridad a los trabajadores y trabajadoras cuando las prestaciones por desempleo son bajas y de insuficiente duración temporal, y hay un bajo nivel de gasto en políticas activas de empleo. Es por este motivo que se hace cada vez más urgente la adopción de una visión más general del mercado de trabajo y su reforma, que tenga en cuenta las interacciones y complementariedades con las políticas sociales y los modelos de bienestar. Este tipo de complementariedades son más fácilmente alcanzables en el marco de un diálogo social permanente y real entre los actores sociales, que permite el diseño de reformas que tengan en cuenta interacciones entre diferentes ámbitos. De hecho, tanto en el caso español como en el caso italiano, las reformas que más se han ajustado al ideal de flexiguridad han sido aquellas resultantes de diálogo social tripartito y pactos sociales.

\section{Bibliografía}

Amable, B. (2003) The Diversity of Modern Capitalism. Oxford: Oxford University Press.

Banco de Italia (2009). Considerazione Finali: Assemblea Ordinaria dei Partecipanti Roma 29 Maggio 2009, Roma: Banca d'Italia. 
Banyuls, J. et alii. (2009). "The transformation of the Employment System in Spain: Towards a Mediterranean Neoliberalism?". En G. Bosch, S. Lehndorff, J. Rubery (eds.) European Employment Models in Flux. A comparison of Institutional Change in Nine European Countries 247-269 New York: Palgrave Macmillan.

Barbier, J. C. (2007). "From political strategy to analytical research and back to politics: a sociological approach of 'flexicurity"'. En J. Jorgensen y P.K. Madsen, Flexicurity and Beyond, (pp. 155-188) Copenhagen: DJØF Publishing...

Baylos, A.; Perez Rey, M. (2006). Sobre el Libro Verde: Moderniza el derecho laboral para afrontar los retos del siglo XXI. Madrid: Fundación Sindical de Estudios.

Berton, F.; Richiardi, M. E ; Sacchi, S. (2009). Flexinsecurity. Perché in Italia la flessibilità diventa precarietà. Bologna: Il Mulino.

Bettio, F. et alii. (2006). "Changes in care regimes and female immigration: the «care drain» in the Mediterranean". Journal of European social policy, Vol 16, Núm. 3, 271-285.

Boeri, T. et alii (2006). The political economy of flexecurity. Madrid: Fundación para el Estudio de la Economía Aplicada (Fedea).

Bredgaard, T.; Larsen, F.; Madsden, K. (2006). "Opportunities and challenges for flexisecurity. The Danish example”. Transfer, Vol. 12, Núm 1, 61-82.

CES (2008) ‘Memoria Sobre la Situación Socioeconómica y Laboral', Madrid: CES.

Comisión Europea (2006). Libro Verde. Modernizar el Derecho laboral para afrontar los retos del siglo XXI. Bruselas: Comisión Europea.

Crouch, C. (2008) The Governance of Labour Market Uncertainty: Towards a New Research Agenda, Hugo Sinzheimer Discussion Paper 2008-08.

Esping-Andersen, G. (2000). Los fundamentos sociales de la economía postindustrial. Barcelona: Ariel.

ETUI (2008). Benchmarking Working Europe 2008. Brussels: ETUI.

European Foundation (2007). Fourth European Working Conditions Survey. Dublin: European Foundation.

European Commission (2004) Employment, Employment, Employment, Brussels: EC. 
EUROSTAT (2007). "Life-long learning (adult participation in education and training." htpp://epp.eurostat.ec.europa.eu.

Ferrera, M. (2005) Welfare State Reform in Southern Europe; Fighting Poverty and Social Exclusion in Italy, Spain, Portugal and Greece, New York: Routledge.

Jepsen, M. (2005) “Towards a gender impact analysis of Flexicurity?" En T. Bredgaard y Bredgaard, T.; Larsen, F. (eds.) (2005) Employment policy from different angles, Copenhagen: Djoef Publishing.

Jørgensen, H.; Madsen, P. K. (eds.) (2007). Flexicurity and Beyond. Copenhagen: DJØF Publishing.

Keune, M.; Jepsen, M. (2007). Not balanced and hardly new: the European Commission's quest for flexicurity. ETUI-REHS, WP 2007.01.

Keune, M.; Pochet, Ph. (2008). "Flexiguridad: utilidad, desventajas y alternativas", Gaceta sindical, nueva etapa, Núm 11: 133-151.

Madsen, P.K. (2004). "The Danish model of flexisecurity". Transfer Review, Vol. 10, Núm 2, 187-208.

Madsen, P.K. (2006): "Labour Market Flexibility and Social Protection in European Welfare States - Contrasts and Similarities", Australian Bulletin of Labour, Vol. 32(2), pp. 139-162.

Martín Artiles, A. (2008). “¿Modelo social europeo de bajo coste?”. Arxius de Ciències Socials, Núm 18, 9-24.

Maurice, M., Sellier, F., Silvestre, J.J. (1987) Política de educación y organización industrial en Francia y en Alemania. Aproximación a un análisis societal, Madrid: MTAS.

Miguélez, F.; Prieto, C. (2009): "Transformaciones del empleo, flexibilidad y relaciones laborales en Europa”. Política y Sociedad, Vol. 46 Núm. 1 y 2, pp. 275-287.

Miguélez, F. (2007) "Flexiseguridad, bienestar y cohesión social". Revista del Ministerio de Trabajo y Asuntos Sociales, Número Extraordinario 1, 145-160.

Molina, Oscar (2005) "Political Exchange and Bargaining Reform in Italy and Spain". European Journal of Industrial Relations, 11(1), 7-26.

Scarpetta, S. (1996) Assessing the Role of Labour Market Policies and Institutional Settings on Unemployment: A Cross-Country Study, OECD Economic Studies n.26. 
Scharpf, F. (1999) Governing in Europe. Effective and Democratic?, Oxford/NY: Oxford University Press.

Seifert, H.; Tangian, A. (2007). "Obstacles for flexisecurity: Precariusness of Flexible Work”. WSI-Hans Boeckler Stifftung, Working Paper, July 2007.

Simonazzi, A. M. (2007). "Care regimes and national employment model". Cambridge Journal of Economics, Núm. 33, 211-232.

Spidla, V. (2005) Is Flexicurity Topping EU Agenda?, http://ec.europa.eu/ employment_social(speeches/2005/vs_140905_en.pdf .

Supiot, A. (2003) Beyond Employment, Oxford/NY: Oxford University Press.

Tangian, A. (2007). "European flexisecurity: concepts, methodology and policies." Transfer, 13(4), 551-573. 